\title{
Robotic-assisted partial Nephrectomy: initial experience in South America
}

\author{
Gustavo C. Lemos, Marcelo Apezzato, Leonardo L. Borges, José R. Colombo Jr \\ Albert Einstein Jewish Hospital (GCL, MA, LLB, JRC jr), and University of São Paulo Medical \\ School (JRC jr), São Paulo, SP, Brazil
}

\begin{abstract}
Objective: To report the initial outcomes of robotic-assisted partial nephrectomy in a tertiary center in South America. Material and methods: From 11/2008 to 12/2009, a total of 16 transperitoneal robotic-assisted partial nephrectomies were performed in 15 patients to treat 18 kidney tumors. One patient with bilateral tumor had two procedures, while two patients with two synchronous unilateral tumors had a single operation to remove them. Eleven (73\%) patients were male and the right kidney was affected in $7(46 \%)$ patients. The median patient age and tumor size were 57 years old and $30 \mathrm{~mm}$, respectively. Five (28\%) tumors were hilar and/or centrally located.

Results: The median operative time, warm ischemia time and estimated blood loss was $140 \mathrm{~min}, 27 \mathrm{~min}$ and $120 \mathrm{~mL}$, respectively. Blood transfusion was required in one patient with bilateral tumor, and one additional pyelolithotomy was performed due to a $15 \mathrm{~mm}$ stone located in the renal pelvis. The histopathology analysis showed 15 ( $83 \%)$ malignant tumors, which $10(67 \%)$ were clear cell carcinoma. The median hospital stay was 72 hrs and no major complication was observed.

Conclusion: Robotic-assisted partial nephrectomy is safe and represents a valuable option to perform minimally invasive nephron-sparing surgery.
\end{abstract}

Key words: kidney; kidney neoplasms; robotics; nephrectomy; treatment outcome

Int Braz J Urol. 2011; 37: 461-467

\section{INTRODUCTION}

With the widespread use of abdominal imaging techniques like ultrasound, CT scan and MRI, the incidence of localized kidney tumor is increasing in a rate of $3 \%$ every year in the last decade (1). Partial nephrectomy is currently the preferred treatment for selected renal masses $<7 \mathrm{~cm}$ whenever is technically feasible, secondary to its oncological equivalency to radical nephrectomy and better functional outcomes $(2,3)$. Additionally, there is robust evidence showing that radical nephrectomy is associated to increased cardiovascular morbidity, hospital admissions, and risk of death compared to partial nephrectomy $(4,5)$.

The minimally invasive approach using the laparoscopic access has gained acceptance and popularity in selected high volume centers and it is now used accordingly to the surgeon's experience, tumor size and location, and patient comorbidities (6). The high technical demanding and steep learning curve of the laparoscopic partial nephrectomy (LPN) are the limitation factors to its larger use in the community setting $(6,7)$. In this scenario, robotic technology is now available to facilitate the learning curve difficulties specially with the reconstruc- 
tive part of the procedure performed under warm ischemia due to its tridimensional magnified view, wristed instruments, tremor filter, completely stable camera handled by the surgeon and better ergonomics (7). This study aims to report the initial outcomes of robotic-assisted partial nephrectomy (RAPN) performed in a tertiary center in South America.

\section{MATERIALS AND METHODS}

From $11 / 2008$ to $12 / 2009,15$ patients had 16 robotic-assisted partial nephrectomies to remove 18 kidney tumors. Data were obtained from our prospectively maintained database with Institutional Review Board approval. Two patients presented with two synchronic unilateral tumors that were removed in a single procedure, while one patient with bilateral tumor had two procedures to avoid the warm ischemia damage in both kidneys at the same time (Figures 1,2 and 3). Eleven $(73 \%)$ patients were male and the right kidney was affected in $7(46 \%)$ patients. The median patient age and tumor size were 57 years (43-73) and 30 $\mathrm{mm}(10-55)$, respectively. Five (28\%) tumors were hilar and/or centrally located. Eight (44\%) tumors were anteriorly located and the remaining $11(66 \%)$ were posterior. The tumors were located in the upper, medial and inferior pole in 50,30, and $20 \%$ of

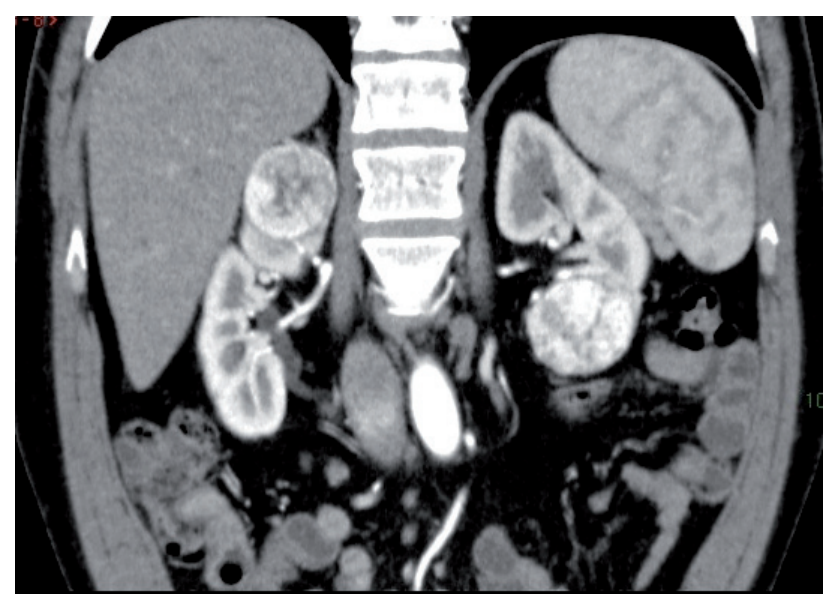

Figure 1 - Patient with bilateral tumors. On the right kidney a $6.2 \mathrm{~cm}$ upper pole lesion is observed, and a $7 \mathrm{~cm} \mathrm{le-}$ sion is noted in the lower pole of the left kidney.
Figures 2 and 3

Follow-up postoperative CT scan images.
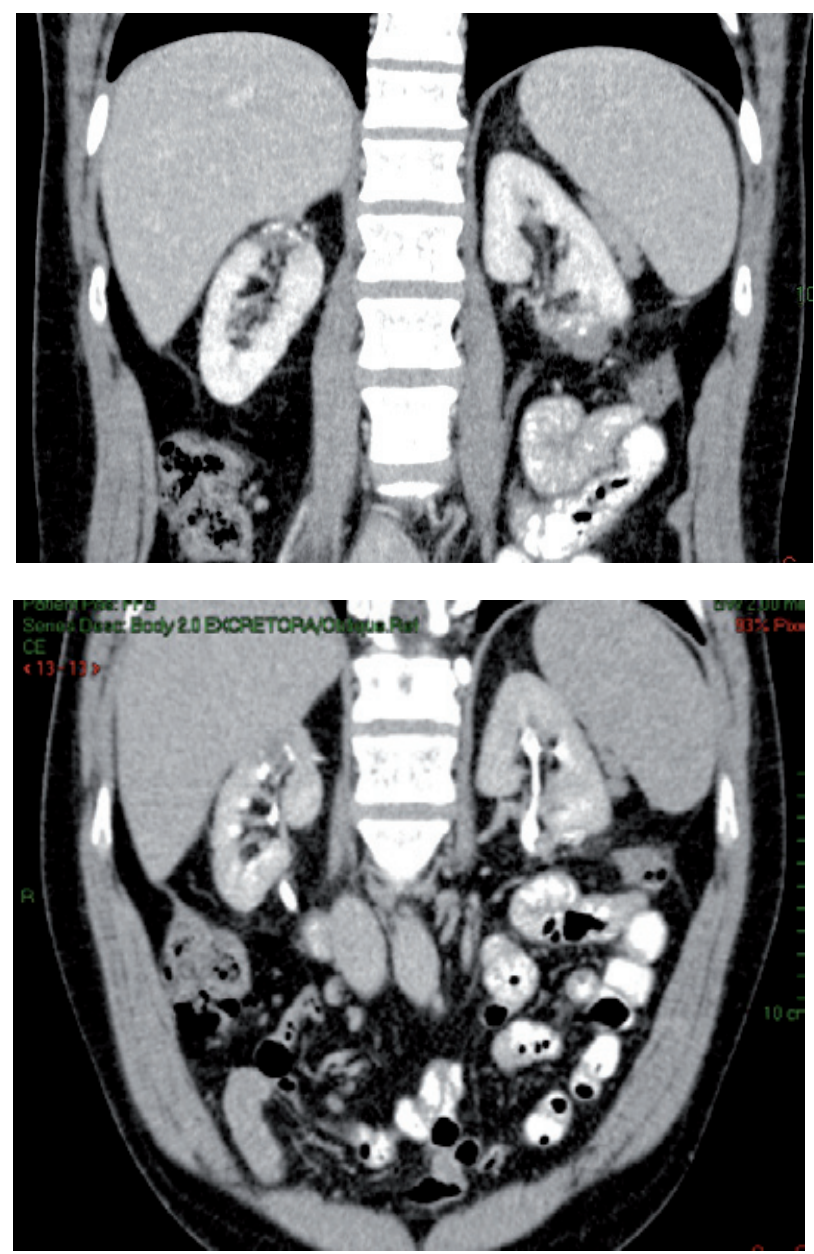

the cases, respectively (Table-1). Tumor excision was performed under warm ischemia in all but one case with a single $10 \mathrm{~mm}$ exophitic tumor.

Briefly, the transperitoneal approach with 3 robotic arms of the da Vinci S surgical System (Intuitive Surgical, Sunnyvale, CA) and one 12 $\mathrm{mm}$ port for the bedside assistant were used. After the colon is mobilized medially, the kidney, ureter and gonadal vein are identified and retracted laterally. The dissection proceeds cephalad along the anterior surface of the psoas muscle until the renal vein is visualized. The renal hilar vessels are carefully dissected individually. The kidney is mobilized within Gerota's fascia and defatted, maintaining perirenal fat over the tumor. Intraoperative flexible laparoscopic ultrasonography is routinely performed to circumferentially score an adequate 
Table 1 - Demographic Data.

\begin{tabular}{|c|c|c|}
\hline & Patients & 15 \\
\hline & Procedures & 16 \\
\hline & Tumors & 18 \\
\hline & Male (\%) & $11(73)$ \\
\hline & Age (yrs) & $57(43-75)$ \\
\hline & Tumor size (mm) & $30(10-55)$ \\
\hline & Right side (\%) & 7 (39) \\
\hline 1 & Central/Hilar (\%) & $5(28)$ \\
\hline & Anterior $(\%)$ & $5(28)$ \\
\hline
\end{tabular}

margin of renal parenchyma. As a nephron-protective measure, $12.5 \mathrm{~g}$ of Mannitol are given intravenously 30 minutes prior to hilar lamping. The renal pedicle vessels are clamped individually with laparoscopic bulldogs, applied by the bedside assistant. The tumor is excised with cold robotic scissors in a near-bloodless field, and the collecting system is suture repaired with a running 2-0 Vicryl on a SH needle. Care should be taken to not place the stitches very deep in order to avoid obstruction to an adjacent collecting system and/ or major arterial branch. Another running suture with Vicryl 0 on CT-1 needle is placed to close the kidney defect. A Hem-o-Lok clip (Weck Closure System, Research Triangle Park, NC) is secured on the suture to prevent it from pulling through, and another clip is applied to the suture with the opposite renal surface, compressing the kidney (sliding clip technique). Mannitol $12.5 \mathrm{~g}$ and furosemide $10-20 \mathrm{mg}$ is given intravenously prior to hilar unclamping. The specimen is extracted in an Endocatch bag. The abdomen is then inspected after 10 minutes of no pneumoperitoneum to check any bleeding from the surgical area. A Blake drain is left in all patients.

\section{RESULTS}

The median operative time in this series was 140 min. (90-170), median estimated blood loss was $120 \mathrm{~mL}(50-1000)$, and median warm ischemia time was 27 min (13-40). Blood transfusion was given to a patient with bilateral tumors after the second procedure. Pathologic analysis revealed 15 (83\%) malignant tumors: 13 renal cell carcinoma (10 clear cell and 3 papillary), and 2 metastatic lung leiomyosarcoma, both in the same patient. All benign lesions were oncocytoma (Table-2).

In one case, after the kidney was reconstructed and the hilum unclamped, the macroscopic aspect of the specimen was suspicious for positive margin, and this finding was confirmed by pathology analysis. Through a small anterior subcostal incision, an additional kidney parenchyma was excised with no additional hilar clamping; instead, the assistant employed manual compression during the parenchymal resection and subsequent reconstruction.

The median hospital stay was 72 hours (36-98), and no perioperative complication was observed in this series. Additionally to the partial nephrectomy, one patient had a robotic-assisted pyelolithotomy due to an ipsilateral $15 \mathrm{~mm}$ stone located in renal pelvis. All patients were followed with the same protocol, and after a median followup of 6.2 months no local or systemic recurrence was observed.

Analyzing the operative outcomes of the RAPN performed for anterior and posterior tumors, we did not find any significant difference regarding 
Table 2 - Perioperative outcomes Pathological results.

\begin{tabular}{lc}
\hline Operative time (min) & $\mathbf{1 4 0}(\mathbf{9 0 - 1 7 0 )}$ \\
\hline Warm ischemia time (min) & $27.5(13-40)$ \\
Estimated blood loss (mL) & $120(50-1000)$ \\
Blood transfusion (\%) & $1(6)$ \\
Positive surgical margin (\%) & $1(6)$ \\
Malignancy (\%) & $15(83)$ \\
RCC $\quad$ Clear cell & 13 \\
$\quad$ Papillary & 10 \\
Leiomyosarcoma & 2 \\
Hospital stay (Hrs) & $72(36-98)$ \\
\hline
\end{tabular}

tumor size ( $3.2 \mathrm{~cm}$ vs. $2.3 \mathrm{~cm} ; \mathrm{p}=0.29)$; operative time (140 min. vs. $122 \mathrm{~min}$; $\mathrm{p}=0.73$ ); warm ischemia time (29 $\mathrm{min}$. vs. $24 \mathrm{~min}$; $\mathrm{p}=0.11$ ), and estimated blood loss (100 mL vs. $122 \mathrm{~mL} ; \mathrm{p}=0.67)$.

\section{DISCUSSION}

Despite the amount of evidence in the literature supporting the nephron sparing surgery, even open partial nephrectomy is proved to be heavily underused in United States, probably because its technical complexity (3). Considering only the laparoscopic approach, the current status is still more dramatic; with only few surgical teams in high volume centers have acquired the advanced skills to execute the procedure safely with a reasonable warm ischemia time $(3,6)$. Adequate kidney dissection with tumor exposure, following by the tumor excision with negative margins and renorrhaphy under hilar clamping are the key steps that have limited the outcomes of LPN compared to the open partial nephrectomy, with longer warm ischemia time and higher postoperative complication rate in a large multicentric study (6). Although recent technical modifications such as early hilar unclamping, suture preplacement, knotless techniques and use of biological sealants have decreased the LPN warm ischemia time and postoperative bleeding, these are only used by surgeons with large experience in the laparoscopic arena (8-11).

The robotic technology incorporated tridimensional intraoperative visualization, better dissection and suture angles, and enhanced ergonomics. It made possible to overcome the steep learning curve of minimally invasive partial nephrectomy, with safe perioperative and oncological results. The presented data showed a warm ischemia time of 27 min., despite the limited experience of the surgeon with LPN ( $<10$ cases); furthermore, almost one third of the treated tumors in this report were hilar and/or centrally located. The median operative time of $140 \mathrm{~min}$. was also comparable to the initial world RAPN series, ranging from 142 to $279 \mathrm{~min}$. (7). Similar findings were seen regarding estimated blood loss, with $120 \mathrm{~mL}$ compared to 92 to $329 \mathrm{~mL}$ in the previous reports (7). Only one patient with bilateral tumor required blood transfusion in our series, and it was necessary after the second procedure, performed two weeks after the resection of the contralateral tumor. 
No major complication was observed in this study. The only open conversion occurred in an elective fashion due to suspicion of macroscopic positive surgical margin in the specimen, after the kidney defect was closed and the renal hilum unclamped. The clinical impact of positive surgical margins after partial nephrectomy is still not well defined, and the available data supporting close follow-up in LPN cases with positive margins is based in microscopic disease found in the final pathology analysis $(12,13)$. While the decision to remove additional renal tissue in this circumstance is controversial, the authors strongly encourage the achievement of negative margins during partial nephrectomy.

The hospital stay for RAPN in the literature has ranged from 48 to 96 hours, what is comparable to the 72 hours presented in this report. The authors usually removed the drain with output $<100 \mathrm{~mL}$ per 12 hours and the patient is then discharged. Although the follow-up in this study is short, no local recurrence or metastatic disease was observed in the patients with renal cell carcinoma.

So far, few studies have compared the pure laparoscopic approach to its robotic counterpart. Wang et al. analyzed 102 cases of minimally invasive partial nephrectomies (40 RAPN and 62 LPN) and concluded that operative time, warm ischemia time and hospital stay were significantly shorter in RAPN series (14). Deane et al. did not find any difference in the results of LPN and RAPN, although this study had a small number of patients (15). Aron et al. showed in a subset of six patients undergone LPN with early hilar unclamping, a significant shorter warm ischemia time compared to RAPN (16). In a multicentric study comparing 118 LPN to 129 RAPN, Benway et al. reported significant advantages to RAPN regarding blood loss (155 vs. $196 \mathrm{~mL}$ ), warm ischemia time (19.7 vs. $28.4 \mathrm{~min}$.), and hospital stay (2.4 vs. 2.7 days) (17).

Although the authors have showed promising results during their learning curve, some limitations of RAPN should be pointed: the presence in the operative team of a laparoscopic skilled bed assistant to apply the bulldogs during hilar control is highly recommended, and the financial costs of the robotic system, surgical supplies, and required medical training represent a serious concern, especially for developing countries.

Randomized studies with larger number of subjects are still awaited to assess the impact of the robotic-assisted nephron-sparing surgery, compared to open and laparoscopic approaches.

\section{CONCLUSIONS}

Robotic-assisted partial nephrectomy was feasible and presented a low complication rate even during the initial learning curve. The data presented herein revealed promising initial perioperative results. The use of the robotic technology may increase the employment of minimally invasive nephron-sparing surgery.

\section{CONFLICT OF INTEREST}

None declared.

\section{REFERENCES}

1. Hock LM, Lynch J, Balaji KC. Increasing incidence of all stages of kidney cancer in the last 2 decades in the United States: an analysis of surveillance, epidemiology and end results program data. J Urol. 2002; 167: 57-60.

2. Thompson RH, Siddiqui S, Lohse CM, Leibovich BC, Russo P, Blute ML. Partial versus radical nephrectomy for 4 to $7 \mathrm{~cm}$ renal cortical tumors. J Urol. 2009; 182: 2601-6.

3. Thompson RH, Kaag M, Vickers A, Kundu S, Bernstein M, Lowrance W, et al. Contemporary use of partial nephrectomy at a tertiary care center in the United States. J Urol. 2009; 181: 993-7.

4. Huang WC, Levey AS, Serio AM, Snyder M, Vickers AJ, Raj GV, et al. Chronic kidney disease after nephrectomy in patients with renal cortical tumours: a retrospective cohort study. Lancet Oncol. 2006; 7: 735-40.

5. Go AS, Chertow GM, Fan D, McCulloch CE, Hsu CY. Chronic kidney disease and the risks of death, cardiovascular events, and hospitalization. N Engl J Med. 2004; 351: 1296-305. Erratum in: N Engl J Med. 2008; 18: 4. 
6. Gill IS, Kavoussi LR, Lane BR, Blute ML, Babineau D, Colombo JR Jr, et al. Comparison of 1,800 laparoscopic and open partial nephrectomies for single renal tumors. J Urol. 2007; 178: 41-6.

7. Gautam G, Benway BM, Bhayani SB, Zorn KC. Robot-assisted partial nephrectomy: current perspectives and future prospects. Urology. 2009; 74: 735-40.

8. Nguyen MM, Gill IS. Halving ischemia time during laparoscopic partial nephrectomy. J Urol. 2008; 179: 627-32; discussion 632.

9. Abaza R, Picard J. A novel technique for laparoscopic or robotic partial nephrectomy: feasibility study. J Endourol. 2008; 22: 1715-9.

10. Cabello JM, Benway BM, Bhayani SB: Robotic-assisted partial nephrectomy: surgical technique using a 3-arm approach and sliding-clip renorrhaphy. Int Braz J Urol. 2009; 35: 199-203; discussion 203-4.

11. Breda A, Stepanian SV, Lam JS, Liao JC, Gill IS, Colombo JR, et al. Use of haemostatic agents and glues during laparoscopic partial nephrectomy: a multi-institutional survey from the United States and Europe of 1347 cases. Eur Urol. 2007; 52: 798-803.

12. Yossepowitch O, Thompson RH, Leibovich BC, Eggener SE, Pettus JA, Kwon ED, et al. Positive surgical margins at partial nephrectomy: predictors and oncological outcomes. J Urol. 2008; 179: 2158-63.

13. Permpongkosol S, Colombo JR Jr, Gill IS, Kavoussi LR. Positive surgical parenchymal margin after laparoscopic partial nephrectomy for renal cell carcinoma: oncological outcomes. J Urol. 2006; 176: 2401-4.

14. Wang AJ, Bhayani SB. Robotic partial nephrectomy versus laparoscopic partial nephrectomy for renal cell carcinoma: single-surgeon analysis of $>100$ consecutive procedures. Urology. 2009; 73: 306-10.

15. Deane LA, Lee HJ, Box GN, Melamud O, Yee DS, Abraham JB, et al. Robotic versus standard laparoscopic partial/wedge nephrectomy: a comparison of intraoperative and perioperative results from a single institution. J Endourol. 2008; 22: 947-52.

16. Aron M, Koenig P, Kaouk JH, Nguyen MM, Desai MM, Gill IS. Robotic and laparoscopic partial nephrectomy: a matched-pair comparison from a high-volume centre. BJU Int. 2008; 102: 86-92.

17. Benway BM, Bhayani SB, Rogers CG, Dulabon LM, Patel MN, Lipkin M, et al. Robot assisted partial nephrectomy versus laparoscopic partial nephrectomy for renal tumors: a multi-institutional analysis of perioperative outcomes. J Urol. 2009; 182: 866-72
Submitted for publication:

July 28, 2010

Accepted after revision:

April 15, 2011

\section{EDITORIAL COMMENT}

The emergence of robotics in urology is an effort to incorporate the minimally invasive benefits of laparoscopy to enhanced vision and increased freedom of movement of the instruments. These advantages help us to perform more complex surgical tasks, such as an accurate surgical closure of collecting system and parenchymal defects.

Although laparoscopic partial nephrectomy is safer in expert hands, laparoscopic partial nephrectomy has a higher complication rate and a significantly longer warm ischemia-time when compared to open approach. To date, open nephron-sparing surgery is the standard of care for T1 renal tumors $(<7 \mathrm{~cm})$ in appropriate patients and laparoscopic radical nephrectomy should be an alternative only if partial resection of $\mathrm{T} 1$ tumors is not technically feasible. Indeed, conventional laparoscopic partial nephrectomy is a challenging procedure with a steep learning curve and it's questionable if a procedure limited to a few experience hands in high volume reference centers to be a viable alternative to open partial nephrectomy. However, in contemporary series from laparoscopic specialized tertiary care centers, several technical modifications of LPN such as early unclamping technique abbreviated the warm ischemia time. 
In the last 6 years, robotic assisted partial nephrectomy (RAPN) has increasingly gained acceptance in robotic centers worldwide with preliminary efficacy and safety data comparable to laparoscopic and open series.

Although, costs of robotic assistance remain an important issue, especially in developing countries with limited health budgets and restricted access to tertiary centers, economic evaluation must be balanced against possible benefits of robot-assistance in reducing ischemia time, complication rate and deleterious short and long-term renal function outcomes, preventing chronic kidney disease.

As show in recent studies, regardless the surgical approach, the duration of warm ischemia should be the minimum as possible, ideally should be completed within 20 minutes. Gradually, the ischemia time has gained importance as a primary perioperative outcome in partial resections of RCC and tumor size seems to be no longer a limiting factor for nephron-sparing surgery.

Although, extended follow-up is lacking, considering ischemic time as a key-point, the initial international RAPN series reported that the learning curve seems to be shorter for robotic than for laparocopy.

In the present study, the authors reported their initial experience with 16 robotic assisted partial nephrectomies. Median warm ischemic time was 27.5 minutes with an interquartile range of 13-40 minutes. Therefore, a high proportion of patients, nearly $50 \%$, were exposed to a warm ischemia time beyond the 30 minutes historical limit. Available literature confirms that the 20 minutes cut-off could be reach after 30 cases.
Despite some methodologic limitations of initial reports (small numbers, limited experience), the authors should be recommended for their pioneering efforts in RAPN in South America.

Some consideration must be given regarding oncologic control. As pointed out by the authors, although only a small portion of patients with positive surgical margin will clinically relapse in short-term follow-up, negative margins are encouraged. As previously demonstrated, nephron-sparing surgery is an oncologic safe procedure. Many efforts have been made to achieve negative margins, prevent local recurrence and survival. Intraoperative laparoscopic ultrasound could be useful in deeper central tumors.

Unlikely, today's robotic platforms have reached their full potential in urology. Technological improvements and operative refinements are expected. Robot assisted nephron-sparing surgery is a promising procedure in evolution, that bridge the advantages of LPN with shorter ischemia time and lower risks of complications. Further studies are needed to confirm this point and if RAPN will be the most effective, economic attractive and safe option for localized renal masses management.

Dr. Flavio L. Heldwein Discipline of Urology Universidade do Sul de Santa Catarina Florianopolis, Brazil E-mail: flavio.lobo@gmail.com

$$
\begin{array}{r}
\text { Dr. Eric Barret } \\
\text { Department of Urology } \\
\text { Institut Mutualiste Montsouris } \\
\text { Paris, France }
\end{array}
$$

